\title{
BOUNDED SLOPE VARIATION AND GENERALIZED CONVEXITY
}

\author{
FRANK N. HUGGINS
}

\begin{abstract}
In this paper, the concept of bounded slope variation, that of the convexity of a function with respect to an increasing function, and the Lebesgue-Stieltjes integral are used to further generalize a theorem of F. Riesz and to give a new proof based on a weaker hypothesis that a function which has bounded slope variation with respect to an increasing function $m$ over $[a, b]$ can be expressed as the difference of two functions each cf which is convex with respect to $m$ on $[a, b]$.
\end{abstract}

Introduction. In [4], F. Riesz proved that a necessary and sufficient condition that a function $F$ defined on the interval $[a, b]$ be the integral of a function of bounded variation on $[a, b]$ is that $F$ have bounded slope variation with respect to $I$ over $[a, b]$, where $I$ is the function defined, for each $x$, by $I(x)=x$. In Theorem 3 of [1], Riesz's result was generalized using the Lane integral with respect to a continuous increasing function $m$ on $[a, b]$ instead of the Riemann integral. In Theorem 1 of this paper, it is shown that by using the Lebesgue-Stieltjes integral, the requirement that $m$ be continuous on $[a, b]$ may be deleted.

A comparison of Definition 1 of this paper with Definitions 3 of [7] and 1 of [6] will reveal that what J. R. Webb and the author call "bounded slope variation with respect to $m$ over $[a, b]$ " is the same concept that A. M. Russell calls "bounded $m$-second variation on $[a, b]$ " and, in case $m(x)=x$ for each $x$, is the same concept that A. W. Roberts and D. E. Varberg call "bounded convexity on $[a, b]$ ". Hence Theorem 2 of this paper generalizes both Theorem 1.1 of [7] and Theorem 3 of [6].

DEFINITION 1 . The statement that $f$ has bounded slope variation with respect to $m$ over $[a, b]$ means that $f$ is a function whose domain includes $[a, b], m$ is a real-valued increasing function on $[a, b]$, and there exists a nonnegative number $B$ such that if $\left\{x_{i}\right\}_{i=0}^{n}$ is a subdivision of $[a, b]$ with $n>1$, then

$$
\sum_{i=1}^{n-1}\left|\frac{f\left(x_{i+1}\right)-f\left(x_{i}\right)}{m\left(x_{i+1}\right)-m\left(x_{i}\right)}-\frac{f\left(x_{i}\right)-f\left(x_{i-1}\right)}{m\left(x_{i}\right)-m\left(x_{i-1}\right)}\right| \leqslant B .
$$

The least such number $B$ is called the slope variation of $f$ with respect to $m$

Received by the editors October 7, 1976.

AMS (MOS) subject classifications (1970). Primary 26A45, 26A51; Secondary 26A42.

Key words and phrases. Bounded slope variation, bounded variation, convex function, Lebesgue-Stieltjes integral 
over $[a, b]$ and is denoted by $V_{a}^{b}(d f / d m)$. [Note. $V_{a}^{a}(d f / d m)=0$.]

The above sum is nondecreasing with respect to refinements.

The name "bounded slope variation" was given to the concept by J. R. Webb in 1960 in his doctoral dissertation which he later published [9]. Theorems and lemmas giving some of the properties of functions which have bounded slope variation with respect to an increasing function on an interval $[a, b]$ may be found in [1], [2], [3], and [9].

By Lemma 3.3 of [9], if $f$ has bounded slope variation with respect to $m$ over $[a, b]$ and $a \leqslant c<b$, then

$$
D_{m}^{+} f(c)=\lim _{x \rightarrow c^{+}} \frac{f(x)-f(c)}{m(x)-m(c)}
$$

exists, and if $a<c \leqslant b$,

$$
D_{m}^{-} f(c)=\lim _{x \rightarrow c^{-}} \frac{f(x)-f(c)}{m(x)-m(c)}
$$

exists.

THEOREM 1. In order that the function $F$ defined on $[a, b]$ be the LebesgueStieltjes integral of a function $f$ of bounded variation on $[a, b]$ with respect to an increasing function $m$ on $[a, b]$, it is necessary and sufficient that $F$ have bounded slope variation with respect to $m$ over $[a, b]$.

Proof. It is easy to see that the condition is necessary. Suppose $F$ has bounded slope variation with respect to $m$ over $[a, b]$. Let $f$ be the function defined, for each $x$ in $[a, b]$, by

$$
\left\{\begin{array}{l}
f(x)=D_{m}^{+} F(x) \quad \text { for each } x \text { in }[a, b), \\
f(b)=D_{m}^{-} F(b) .
\end{array}\right.
$$

By Corollary 8.1 of [2], $f$ is of bounded variation on $[a, b]$. By Theorem 6 of [2], $F$ is absolutely continuous with respect to $m$ on $[a, b]$. Hence $F$ is the indefinite Lebesgue-Stieltjes integral on $[a, b]$ of a function $g$ with respect to $m[5$, p. 127]. However, any Lebesgue-Stieltjes integral $F$ on $[a, b]$ with respect to an increasing function $m$ is the Lebesgue-Stieltjes integral on $[a, b]$ of any one of the right-hand (left-hand) derivates of $F$ with respect to $m$ [8, p. 59]. Therefore we have $F(x)=\int_{a}^{x} f d m+F(a)$ for each $x$ in $[a, b]$. That is, $F$ is the Lebesgue-Stieltjes integral of a function $f$ of bounded variation on $[a, b]$ with respect to an increasing function $m$ over $[a, b]$.

It should be noted that if $m=I$, the Lebesgue-Stieltjes integral reduces to the ordinary Lebesgue integral and, since $f$ is of bounded variation on $[a, b]$, the Riemann integral $\int_{a}^{x} f d x, x$ in $[a, b]$, is equal to the Lebesgue integral of $f$ over $[a, x]$ so that Riesz's theorem is a special case of Theorem 1 .

Definition 2. The statement that $f$ is convex with respect to $m$ on $[a, b]$ means that $f$ is a real-valued function whose domain includes $[a, b], m$ is a real-valued increasing function on $[a, b]$, and if $x_{1}$ and $x_{2}$ are numbers in $[a, b]$ with $x_{1}<x_{2}$, then $f(x) \leqslant \varphi(x)$ for each $x$ in $\left(x_{1}, x_{2}\right)$, where 


$$
\varphi(x)=\frac{f\left(x_{2}\right)-f\left(x_{1}\right)}{m\left(x_{2}\right)-m\left(x_{1}\right)} m(x)+\frac{f\left(x_{1}\right) m\left(x_{2}\right)-f\left(x_{2}\right) m\left(x_{1}\right)}{m\left(x_{2}\right)-m\left(x_{1}\right)} .
$$

$\varphi$ is called the $m$-chord of $f$ through the points $\left(x_{1}, f\left(x_{1}\right)\right)$ and $\left(x_{2}, f\left(x_{2}\right)\right)$. [Note that $\varphi\left(x_{1}\right)=f\left(x_{1}\right)$ and $\varphi\left(x_{2}\right)=f\left(x_{2}\right)$.] Interpreted geometrically, the statement that $f$ is convex with respect to $m$ on $[a, b]$ means that if $x_{1}$ and $x_{2}$ are numbers in $[a, b]$ with $x_{1}<x_{2}$, every point on the curve $y=f(x)$ between $\left(x_{1}, f\left(x_{1}\right)\right)$ and $\left(x_{2}, f\left(x_{2}\right)\right)$ lies on or below the $m$-chord of $f$ through $\left(x_{1}, f\left(x_{1}\right)\right)$ and $\left(x_{2}, f\left(x_{2}\right)\right)$. In case $m(x)=x$ for each $x$, Definition 2 reduces to the usual definition of a convex function.

It should be noted that a function which has bounded slope variation with respect to $m$ over $[a, b]$ is not necessarily convex with respect to $m$ on $[a, b]$. For example, $I^{3}$ has bounded slope variation with respect to $I$ on $[-1,1]$ (Corollary 3.1 of [2]), but $I^{3}$ is not convex with respect to $I$ on $[-1,1]$. On the other hand, a function which is convex with respect to $m$ on $[a, b]$ does not necessarily have bounded slope variation with respect to $m$ over $[a, b]$. For example, $-I^{1 / 2}$ is convex with respect to $I$ on $[0,1]$, but $-I^{1 / 2}$ does not have bounded slope variation with respect to $I$ on $[0,1]$. However, it can be shown that if $f$ is convex with respect to $m$ on $[a, b]$, then $f$ has bounded slope variation with respect to $m$ over $[a, b]$ if and only if both $D_{m}^{+} f(a)$ and $D_{m}^{-} f(b)$ exist. Moreover, in this case we have that $V_{a}^{b}(d f / d m)=D_{m}^{-} f(b)-D_{m}^{+} f(a)$.

Lemma 1. If $F$ is the Stieltjes, mean Stieltjes, Lane, or Lebesgue-Stieltjes integral of a nondecreasing function $f$ with respect to an increasing function $m$ on $[a, b]$, then $F$ is convex with respect to $m$ on $[a, b]$.

Proof. If $x_{1}$ and $x_{2}$ are numbers in $[a, b]$ with $x_{1}<x_{2}$ and $x$ is a number in $\left(x_{1}, x_{2}\right)$, then

$$
\begin{aligned}
\varphi(x)-F(x)= & \frac{F\left(x_{2}\right)-F\left(x_{1}\right)}{m\left(x_{2}\right)-m\left(x_{1}\right)} m(x) \\
& +\frac{F\left(x_{1}\right) m\left(x_{2}\right)-F\left(x_{2}\right) m\left(x_{1}\right)}{m\left(x_{2}\right)-m\left(x_{1}\right)}-F(x) \\
= & \frac{m(x)-m\left(x_{1}\right)}{m\left(x_{2}\right)-m\left(x_{1}\right)}\left[F\left(x_{2}\right)-F(x)\right] \\
& -\frac{m\left(x_{2}\right)-m(x)}{m\left(x_{2}\right)-m\left(x_{1}\right)}\left[F(x)-F\left(x_{1}\right)\right],
\end{aligned}
$$

and, since $F(x)=\int_{a}^{x} f d m+F(a)$ for each $x$ in $[a, b]$ where $f$ is nondecreasing on $[a, b]$ and $m$ is increasing on $[a, b]$,

$$
F\left(x_{2}\right)-F(x)=\int_{x}^{x_{2}} f d m \geqslant f(x)\left[m\left(x_{2}\right)-m(x)\right],
$$

and

$$
F(x)-F\left(x_{1}\right)=\int_{x_{1}}^{x} f d m \leqslant f(x)\left[m(x)-m\left(x_{1}\right)\right]
$$


so that

$$
\begin{aligned}
\varphi(x)-F(x) \geqslant & \frac{m(x)-m\left(x_{1}\right)}{m\left(x_{2}\right)-m\left(x_{1}\right)} f(x)\left[m\left(x_{2}\right)-m(x)\right] \\
& -\frac{m\left(x_{2}\right)-m(x)}{m\left(x_{2}\right)-m\left(x_{1}\right)} f(x)\left[m(x)-m\left(x_{1}\right)\right]=0 .
\end{aligned}
$$

That is, $F(x) \leqslant \varphi(x)$. Therefore $F$ is convex with respect to $m$ on $[a, b]$.

THEOREM 2. If $F$ is a function whose domain includes $[a, b]$ and $m$ is an increasing function on $[a, b]$, then in order that $F$ have bounded slope variation with respect to $m$ over $[a, b]$, it is necessary and sufficient that $F=G-H$ on $[a, b]$ where each of $G$ and $H$ is convex with respect to $m$ on $[a, b]$ and has bounded slope variation with respect to $m$ over $[a, b]$.

Proof. By Theorem 1, if $F$ has bounded slope variation with respect to $m$ over $[a, b]$, then

$$
F(x)=\int_{a}^{x} f d m+F(a)
$$

for each $x$ in $[a, b]$ where $f$ is of bounded variation on $[a, b]$ and the integral is the Lebesgue-Stieltjes integral. Being of bounded variation on $[a, b]$, $f=g-h$ on $[a, b]$ where each of $g$ and $h$ is nondecreasing on $[a, b]$. Consequently,

$$
F(x)=\int_{a}^{x} f d m+F(a)=\int_{a}^{x} g d m-\int_{a}^{x} h d m+F(a)
$$

for each $x$ in $[a, b]$. Let

$$
G(x)=\int_{a}^{x} g d m+F(a) ; \quad H(x)=\int_{a}^{x} h d m
$$

for each $x$ in $[a, b]$. Then $F=G-H$ on $[a, b]$ where, by Lemma 1, each of $G$ and $H$ is convex with respect to $m$ on $[a, b]$. Moreover, being the Lebesgue-Stieltjes integral of a function of bounded variation with respect to an increasing function $m$ on $[a, b]$, each of $G$ and $H$ has bounded slope variation with respect to $m$ over $[a, b]$ by Theorem 1 .

If $F=G-H$ on $[a, b]$ where each of $G$ and $H$ is convex with respect to $m$ on $[a, b]$ and has bounded slope variation with respect to $m$ over $[a, b]$, then $F$, being the difference of two functions each having bounded slope variation with respect to $m$ over $[a, b]$, is a function having bounded slope variation with respect to $m$ over $[a, b]$.

\section{REFERENCES}

1. F. N. Huggins, A generalization of a theorem of F. Riesz, Pacific J. Math. 39 (1971), 695-701.

2. , Bounded slope variation, Texas J. Sci. 24 (1973), 431-437.

3. ___ Generalized Lipschitz conditions, Texas J. Sci. 27 (1976), 251-256.

4. F. Riesz, Sur certains systèmes singuliers d'équations intégrales, Ann. Ecole Norm. Sup. (3) 28 (1911), 33-62.

5. F. Riesz and B. Sz.-Nagy, Functional analysis, 2nd ed., Ungar, New York, 1953. 
6. A. W. Roberts and D. E. Varberg, Functions of bounded convexity, Bull. Amer. Math. Soc. 75 (1969), 568-572.

7. A. M. Russell, Functions of bounded second variation and Stieltjes-type integrals, J. London Math. Soc. (2) 2 (1970), 193-208.

8. W. H. Young, On integrals and derivates with respect to a function, Proc. London Math. Soc. (2) 15 (1916), 35-63.

9. J. R. Webb, A Hellinger integral representation for bounded linear functionals, Pacific J. Math. 20 (1967), 327-337.

Department of Mathematics, University of Texas, Arlington, Texus 76019 